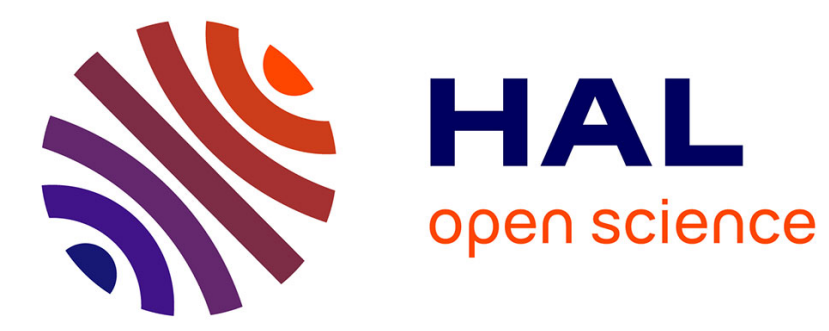

\title{
Le patrimoine européen dans les guides touristiques de mémoire
}

Stefano Vicari

\section{To cite this version:}

Stefano Vicari. Le patrimoine européen dans les guides touristiques de mémoire. Culture et Musées, 2019, 33, pp.87-110. 10.4000/culturemusees.2916 . hal-03125866

\section{HAL Id: hal-03125866 \\ https://hal.science/hal-03125866}

Submitted on 29 Jan 2021

HAL is a multi-disciplinary open access archive for the deposit and dissemination of scientific research documents, whether they are published or not. The documents may come from teaching and research institutions in France or abroad, or from public or private research centers.
L'archive ouverte pluridisciplinaire HAL, est destinée au dépôt et à la diffusion de documents scientifiques de niveau recherche, publiés ou non, émanant des établissements d'enseignement et de recherche français ou étrangers, des laboratoires publics ou privés. 


\section{Le patrimoine européen dans les guides touristiques de mémoire Stefano Vicari Université de Gênes}

\section{Introduction}

Le centenaire de la Première Guerre mondiale a fait l'objet, surtout en France, d'un grand nombre de manifestations commémoratives, éducatives, scientifiques et institutionnelles ${ }^{1}$ témoignant d'un véritable engouement des Français pour leur histoire, notamment pour les deux guerres mondiales. Les lieux où se sont déroulés des événements marquants des deux conflits sont désormais considérés comme partie intégrante du patrimoine touristique français et sont devenus des destinations contribuant au développement de ce qu'on appelle désormais le tourisme de mémoire. En 2012, les ministères de la Défense et du Tourisme estiment que ce tourisme a atteint un chiffre d'affaires de 45 millions d'euros, et que $45 \%$ de la fréquentation des sites de mémoire est représentée par une clientèle internationale, d'origine plutôt européenne (ministères de la Défense et du Tourisme, 2012). L'Agence de développement touristique

1. Voir le site https://www.centenaire.org/fr pour un aperçu des projets et des manifestations liés au centenaire de la Première Guerre mondiale (consulté le 16 octobre 2019). 
de la France (Atout France) le considère aujourd'hui comme un enjeu majeur pour le tourisme en Europe. C'est dans le cadre de cet intérêt renouvelé pour les passés troublés que la maison d'édition Michelin décide de republier six guides touristiques présentant les champs de bataille français d'autrefois et que Le Routard et Le Petit Futé se lancent dans l'édition de guides consacrés à la découverte des lieux symboles de l'histoire française et européenne.

Dans cette étude, qui s'inscrit dans la perspective méthodologique française de l'analyse de disours (Mainguenau \& Chareaudeau, 2002), après la présentation du tourisme de mémoire et des enjeux dont il serait porteur dans le discours des institutions, j'analyserai les procédés discursifs contribuant non seulement à la construction d'un discours patrimonial de mémoire, mais aussi à l'inscription éventuelle de ce patrimoine mémoriel dans une dimension européenne, telle qu'elle est souhaitée par les institutions engagées dans le développement de ce type de tourisme.

\section{Le tourisme de mémoire et les lieux de mémoire : définitions, enjeux et objectifs}

\section{Définitions et objectifs}

Considéré par les ministères du Tourisme et de la Défense comme une sous-catégorie du tourisme culturel, le tourisme de mémoire, tel qu'il s'est développé en tant que filière touristique de masse à partir des années 2000, est défini comme "toute pratique de valorisation et de fréquentation de sites associés aux guerres, violences ou passés traumatiques " (Hertzog, 2013 : 53), et, notamment, des lieux des bataille des deux conflits mondiaux, dont la France détient le plus riche patrimoine en Europe. Les enjeux du tourisme de mémoire dépassent largement les questions purement économiques et de développement du territoire : les acteurs institutionnels et muséaux impliqués dans ce type de tourisme ont l'objectif de participer de façon active "à la construction de l'identité du territoire, de la Nation, de l'Europe " (Urbain, 2003 : 5) tout en s'appuyant sur le sentiment 
d'appartenance et la volonté d'adhésion des populations locales.

Dans les textes institutionnels, toute une rhétorique de la paix et de la coopération internationale est développée, dans le cadre d'une politique mémorielle fondée sur la promotion de commémorations communes et sur les systèmes éducatifs nationaux européens :

"Les Rencontres européennes de la mémoire, organisées le 10 novembre 2008, lors de la célébration de la fin de la Première Guerre mondiale, ont permis de poser les bases d'une politique de mémoire à l'échelon européen. À cette occasion, les représentants des pays de l'Union européenne se sont engagés à mettre en place une présentation et une mise en valeur du patrimoine mémoriel de la Grande Guerre avec, en particulier, la mise en commun d'éléments des archives de guerre à travers la création d'un portail mémoriel européen. A enfin été abordée la question de la transmission de la mémoire et de l'histoire de la Première Guerre mondiale à travers les systèmes éducatifs nationaux.

Depuis le début de l'année 2014, les commémorations liées au centenaire de la Grande Guerre et au $70^{\mathrm{e}}$ anniversaire de la Deuxième Guerre mondiale ont donné lieu à de nombreuses cérémonies internationales ${ }^{2}$."

Les topoï du devoir de mémoire (Ledoux, 2016), de patrimoine commun européen, de mémoire collective (Halbwachs, 1967), sont alors fréquemment convoqués dans les discours institutionnels circulant autour du tourisme de mémoire, qui est considéré comme un vecteur de la construction identitaire européenne, capable, aux yeux des institutions, de réactiver des synergies entre histoire nationale, armée et éducation à la citoyenneté. C'est ainsi qu'on peut lire dans la convention nationale entre le ministère du Tourisme et le secrétariat d'État aux Anciens Combattants : "Dans une période troublée par les grands événements internationaux, le tourisme de mémoire apparaît comme un vecteur de paix, un vecteur d'échange et de respect mutuel entre les peuples " (Bertrand, 2004). Le tourisme de mémoire, selon les acteurs institutionnels engagés

2. Site du ministère de la Défense, en ligne : https://www.defense.gouv.fr/memoire/memoire/tourisme-de-memoire-et-memoire-partagee/memoire-partagee (consulté le 16 octobre 2019). 
dans ce type de tourisme, s'inscrit donc dans une dimension foncièrement européenne : il permettrait de réactiver et de développer des enjeux identitaires supranationaux contribuant à la construction et au renforcement, auprès de la population, du sentiment d'appartenance à la communauté européenne, et cela à partir de la mise en tourisme des lieux de mémoire.

\section{Des lieux des guerres aux lieux de mémoire : tourisme de mémoire et valeurs européennes}

À partir des années 2000, les lieux portant les traces des deux guerres mondiales (parmi d'autres, le Chemin des Dames, Verdun, etc.) font l'objet d'une mise en tourisme par la création - de la part des collectivités territoriales, de la Direction de la mémoire, du patrimoine et des archives (DMPA), et, enfin, du ministère du Tourisme - de musées, monuments, mémoriaux, qui ponctuent désormais les paysages français actuels. C'est ainsi que les lieux des guerres deviennent "lieux de mémoire " (Nora, 1984) à sauvegarder contre le risque d'oubli : " un objet devient lieu de mémoire quand il échappe à l'oubli, par exemple avec l'apposition de plaques commémoratives, et quand une collectivité le réinvestit de son affect et de ses émotions " (Nora, 1984: XvII). Ces lieux font donc l'objet d'un processus de patrimonialisation tant au niveau des actions de valorisation entreprises par les différents acteurs, qu'au niveau des discours qui circulent autour d'eux :

"Si certains lieux de mémoire sont érigés en symboles de
réconciliation franco-allemande dès les années 1960 dans
le contexte de la construction européenne (Chemin des
Dames), le thème de la "mémoire partagée" est un registre
de plus en plus convoqué dans les processus de mise en
tourisme contemporains. La fonction pacificatrice du tou-
risme de mémoire nourrit donc l'imaginaire touristique et
innerve les discours tant au niveau de l'État qu'au niveau
local "(Hertzog, 2012).

Investis d'une large part de symbolique, ces lieux sont censés participer à la construction de l'identité collective des Français et des Européens, et sont devenus partie intégrante du patrimoine non seulement national, mais aussi euro- 
péen, comme le montre bien la présentation du Mémorial de Rivesaltes, publiée sur le site institutionnel :

\begin{abstract}
"D'autres manifestations culturelles - les Journées européennes du patrimoine ou la Semaine nationale de la lutte contre le racisme et l'antisémitisme - constituent autant d'occasions d'associer les établissements scolaires lors de visites, conférences, tables rondes ou encore spectacles. Des "Rencontres de jeunes Européens" sont également organisées chaque année. Toutes ces activités qui associent les jeunes participent au dialogue intergénérationnel et font partager le message humaniste du mémorial. "3
\end{abstract}

Si du point de vue institutionnel, la promotion de la politique touristique du patrimoine mémoriel se fait par l'inscription d'une dimension collective européenne, qu'en est-il de cette dimension dans les guides touristiques de mémoire ? Comment et dans quels buts ces guides, qui émanent de maisons d'édition privées françaises et qui s'adressent principalement à un public francophone, participent de la construction d'un discours patrimonial européen ? Est-ce que cette dimension se construit différemment selon les différentes typologies auxquelles appartiennent les guides analysés? Après la présentation du corpus, je m'attacherai à montrer les procédés discursifs à travers lesquels se construit la dimension européenne du patrimoine mémoriel dans ces guides.

\title{
Les guides touristiques de mémoire
}

Le corpus est constitué de six guides Michelin, de trois guides Le Routard et d'un guide Petit Futé consacrés à la découverte des lieux de mémoire en France : homogènes du point de vue des contenus et de la période de publication, ces guides sont plutôt hétérogènes du point de vue de leur typologie et du public ciblé par chacun.

Publiés entre 2011 et 2016, les six guides touristiques Michelin Les Champs de bataille - La Marne et la

3. En ligne : http://histoires-du-roussillon.eklablog.com/rivesaltes-c28680522 (consulté le 16 octobre 2019). 
Champagne (2011, abrégé en MMC) ; Verdun, Argonne, Saint-Mihiel (2011, abrégé en MVAS) ; Alsace Moselle : Les combats des Vosges (2012, abrégé en MAM) ; Flandres Artois : Ypres, Nord, Pas-de-Calais (2013, abrégé en MFA); Le Chemin des Dames: Aine, Oise (2014, abrégé en MCD) ; Somme:Amiens, Péronne, Albert (2016, abrégé en MS) constituent une réédition revue et abrégée d'une collection de vingt-neuf guides déjà publiés entre 1917 et 1922 par la même maison d'édition. Les guides actuels abandonnent les tons nationalistes et germanophobes innervant le discours historiographique de l'après-guerre (Kottelat, 2015) pour adopter un discours qui se veut plus objectif et impartial, soucieux de récupérer une mémoire commune européenne du conflit mondial, au-delà des clivages d'autrefois. Adressés à un public plutôt cultivé, passionné d'histoire et de littérature (voir les nombreuses citations littéraires des écrivains de la Grande Guerre), et cultivant un certain goût pour l'érudition, ces guides se caractérisent par une superposition de différentes strates énonciatives (ibid.), correspondant - du moins partiellement - aux sections des guides.

La partie introductive ("Comprendre la Grande Guerre ") est signée par François Cochet, historien spécialiste de la Grande Guerre. Les parties qui suivent, distinctes pour chaque guide, sont signées elles aussi par d'autres historiens et visent à renseigner le lecteur sur le conflit dans les régions concernées par le guide. Le récit des événements liés aux lieux décrits ("Découvrir les champs de bataille ") est en revanche attribuable à l'équipe éditoriale et se caractérise par une forte "hybridation du discours historiographique avec le discours touristique "(ibid. : 268) typique de tout guide touristique (Kerbrat-Orecchioni, 2004 ; Margarito, 2004).

La reprise dans des encadrés des "Journaux des marches et opérations " (JMO), et, enfin, la publication d'extraits des anciens guides, par rapport auxquels les rédacteurs n'hésitent pas à prendre leurs distances et à signaler des erreurs éventuelles, ponctuent le texte à plusieurs reprises et constituent l'intertexte sur lequel se forge le discours des guides actuels. Dans les parties de récit historique, le souci d'objectivité et de précision du discours historiographique 
se manifeste par l'adoption d'un régime énonciatif où les marques de subjectivité sont très rares et par la mise en discours de renseignements très détaillés sur les faits historiques relatés, sans hésiter à puiser dans le lexique de spécialité :

"Le musée occupe les bâtiments d'un ancien couvent $\left(17^{\mathrm{e}} \mathrm{s}\right.$.), un grenier du $15^{\mathrm{e}} \mathrm{s}$. et plusieurs salles qui relient ou prolongent cet ensemble; dans les sous-sols sont conservés des vestiges in situ de thermes gallo-romains. [...] La vie quotidienne à l'époque gallo-romaine de même que celle de l'époque mérovingienne occupent une place importante : tombes, bijoux, vaisselle. Un ensemble de l'époque paléochrétienne s'articule autour du chancel de St-Pierre-aux-Nonnains. L'époque médiévale évoque elle aussi la vie quotidienne; de magnifiques plafonds peints à la détrempe au $13^{\mathrm{e}} \mathrm{s}$. présentent des médaillons au décor fantastique. Les beaux-arts sont illustrés par des tableaux des écoles françaises du $19^{\mathrm{e}} \mathrm{s}$. : Corot, Delacroix, Moreau, et des peintres de l'école de Metz (1834-1870) " (MAM, 2012 : 64).

Conçus idéalement pour un public plus jeune, sans doute moins intéressé par l'approfondissement de certains aspects de l'histoire culturelle du pays, et plus soucieux d'informations pratiques prêtes à l'emploi, les trois guides de mémoire Le Routard - Picardie 14-18. Centenaire d'un conflit mondial $(2013, R P)$; Le Débarquement \& la bataille de Normandie (2014, RD) ; Grande Guerre 14-18. Les chemins de mémoire. Des Flandres aux Vosges (2015, RGG) accompagnent le lecteur à la découverte des lieux de mémoire à partir d'une présentation chronologique des événements liés à ces lieux. Les guides s'ouvrent tous les trois avec une préface d'une page signée soit par des représentants des institutions et de structures touristiques du tourisme de mémoire, soit par un historien (Nicolas Offenstadt, $R G G$ ), qui garantissent les contenus des guides tout autant que leur intérêt pour la visite. Si dans le $R G G$, la partie introductive historiographique est plutôt développée (elle s'étend sur cinq pages signées par un autre historien, François Viltart), dans les deux autres, elle ne fait pas l'objet d'une section à part : le discours historiographique est intégré à la section de découverte des lieux, l'hybridation entre discours promotionnel touristique et discours historiogra- 
phique étant dès lors plus évidente que dans les guides Michelin. À cet effet, contribuent également l'emploi, dans les titres et sous-titres, d'une large palette de couleurs, l'affichage d'une nature dialogale, reproduisant l'oralité des échanges ordinaires et réduisant, par là même, la distance entre lecteurs et énonciateurs ("Encore plus ! ", RD, 2014 : 21), et, enfin, la présence de jugements de valeur par rapport aux événements historiques ("Injuste retour des choses ", RP, 2013 : 35). Si les citations de personnages historiques ne sont pas absentes, la place faite aux citations littéraires et à la terminologie technique est presque nulle : dans les descriptions des sites, les rédacteurs adoptent un lexique moins spécialisé, moulé dans des structures syntaxiques volontiers oralisées, avec le recours presque systématique à une ponctuation expressive (points de suspension, point d'exclamation, etc.) interpellant les lecteurs, auxquels les auteurs relatent le ressenti :

"En 2013, on a ainsi réussi à identifier Albert Dadure, un
conscrit de 21 ans originaire de la Manche, mort le
7 février $1915 \ldots$ Tout autour, la nuée de trous d'obus et les
deux entonnoirs de mine ( 6 t d'explosif !) disent bien la
violence des combats. À l'arrière, un poste de secours voi-
sine avec des rails étroits où circulaient des wagonnets
chargés de munitions. L'environnement possède toujours
une forte charge émotionnelle, ici le paysage "parle"... "
(RGG, 2015 : 133).

Le Guide des lieux de mémoire du Petit Futé (2016, PF) se situe davantage dans la lignée des guides dits " pratiques ", tels que les Routard, que dans celle des guides culturels Michelin, et présente les lieux de mémoire français et d'outre-mer depuis la Révolution jusqu'aux deux guerres mondiales. Suite à une première partie d'introduction aux évènements historiques, non signée, le guide introduit les lieux région par région, classées par ordre alphabétique. Du point de vue discursif, il se caractérise par le recours massif au lexique des émotions, dont les autres guides ne sont pas non plus exempts. Cela est vrai tant dans les didascalies des rares images des lieux de mémoire ("Étonnantes traces laissées par le fort de Mutzig dans le paysage ", $P F, 2016$ : 52) que dans le corps du texte ("Une visite passionnante incontournable dans la région ", $P F, 54$; 
"Le système de défense allemand et les vestiges des tranchées françaises sont également visibles sur le site, émouvants témoins de la guerre des tranchées ", $P F, 59)$.

Si tous les guides obéissent aux impératifs de séduction, d'invitation au voyage (Kerbrat-Orecchioni, 2004 : 132), de transmission de connaissances (Moirand, 2004) et d'accompagnement des touristes potentiels dans les lieux de mémoire (Margarito, 2004), le poids de chacune de ces fonctions dans les différentes typologies des guides pris en compte change sensiblement, ce qui a des retombées sur les stratégies typographiques, textuelles et discursives adoptées en fonction des publics respectifs.

\section{La construction discursive du patrimoine européen dans les guides touristiques de mémoire}

Comme l'a très bien montré Gellereau (2011), la valorisation de la mémoire collective de la Grande Guerre passe principalement par des discours contribuant à la construction d'une "biographie culturelle "(ibid. : 75) des objets de guerre exposés dans les différents musées qui leur sont consacrés. Ce serait donc la mise en récit qui permet de reconfigurer ces objets et, par là, de déclencher un processus de patrimonialisation du passé via les objets-signes qui en constituent les traces aujourd'hui. Du point de vue discursif, le patrimoine peut donc bien être considéré à la fois comme " un dispositif social et un discours organisateur de la mémoire d'un groupe, dans un contexte historique et social donné. C'est un ensemble d'images et de représentations, de décisions terminologiques ("ceci est patrimonial ") et juridiques (les inscriptions sur les listes officielles)" (Paveau, 2009 : 36). Dans ces guides, ce dispositif est construit à partir de différentes strates discursives se superposant, du moins partiellement, et contribuant à la patrimonialisation des lieux de mémoire en tant que symboles de la construction européenne.

La première strate : la dimension didactique 
Comme l'affirme Urbain (2003), le tourisme de mémoire se présente comme un "tourisme du présent en quête de synchronie avec le monde contemporain ". C'est bien ce qui arrive pour les "passés troublés " que les guides se proposent de faire découvrir aux visiteurs potentiels : les parties introductives (préfaces et introductions) du Routard et du Petit Futé tiennent un discours explicite sur la nécessité, pour les Européens, de se souvenir de certains événements ayant contribué à forger l'Europe d'aujourd'hui et les valeurs sur lesquelles celle-ci est censée reposer :

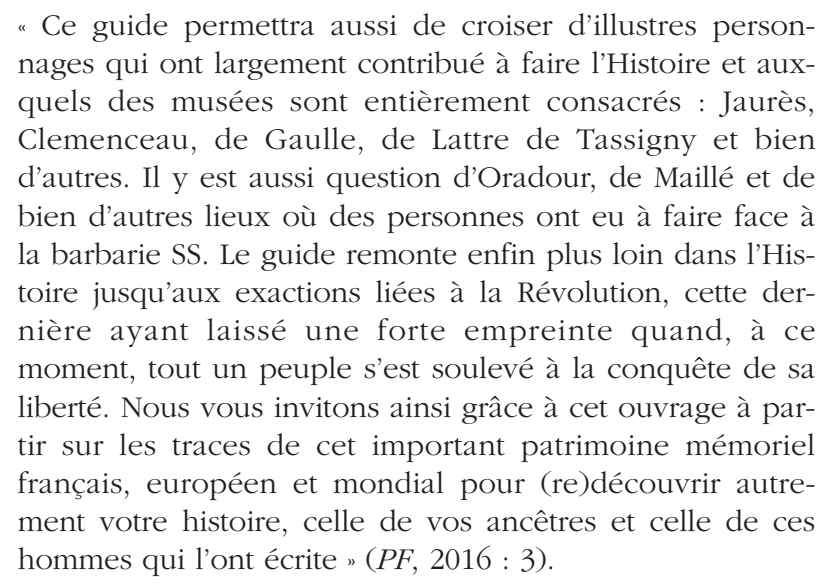

Les " illustres personnages " ayant forgé l'" Histoire " française et européenne, le rappel de la Révolution et des valeurs de liberté dont elle est porteuse, confluent dans la dénomination anaphorique infidèle (Kleiber, 1986) " cet important patrimoine mémoriel " à valeur, pourrait-on dire, résomptive ou synthétisante, en ce qu'elle englobe dans un seul syntagme les différents aspects exposés précédemment dans le texte et inscrit de manière explicite en discours la dimension patrimoniale. Le climax d'adjectifs (" français, européen et mondial ") ancre les événements ainsi patrimonialisés dans les dimensions nationale, européenne, voire universelle, et accompagne le passage de l'" Histoire " à la dimension individuelle, voire communautaire, scellée par l'emploi du possessif (" votre histoire ", "vos ancêtres "). Il en va de même pour la préface et l'introduction du $R G G$, signées par deux historiens garantissant la qualité du discours produit dans le guide : 
" La Grande guerre [...] interroge à la fois le passé et le présent. Un présent hérité des grands drames du $\mathrm{XX}^{\mathrm{e}}$ siècle qui nous amène à réfléchir sans cesse à la construction européenne, au rôle de l'État, à la place de l'individu, au sens de la modernité et surtout à la nécessité de maintenir la paix dans le monde "( $R G G, 2015$ : "Introduction ", 9).

"L'histoire, comme connaissance du passé, ne s'apprend pas seulement dans les livres ou à l'école. Se déplacer sur les lieux, arpenter les traces qui demeurent visibles, regarder le présent pour saisir le feuilletage du temps qui le compose, voilà aussi un apprentissage historique autant qu'un riche moment personnel. Il faut bien se convaincre que "l'histoire de plein air" et la déambulation sur les sites et lieux de mémoire sont un enjeu central pour la transmission et la compréhension du passé dans nos sociétés du XXI e siècle " $(R G G, 2015$ : "Préface ", 7).

Sous la plume des deux historiens, la " transmission et la compréhension du passé ", et l'invitation à réfléchir "sans cesse " prennent la forme d'impératifs moraux et catégoriques (" nécessité ", "Il faut bien se convaincre ", infinitivisations, emploi de "voilà ", énoncés constatifs), et participent du processus de patrimonialisation de ce passé européen, tant il est vrai que "l'obligation de transmettre aux générations futures " constitue l'un des " gestes " de la patrimonialisation (Davallon, 2006 : 126). Si, en effet, ces obligations vont sans dire, c'est parce qu'elles reposent de manière tacite sur le risque d'oubli et de perte de mémoire qui sous-tend tout discours patrimonial, et sur l'idée que " plus le patrimoine est menacé de destruction, plus sa valeur augmente " (Kott, 2013 : 119), ce qui fait écho au " devoir de mémoire ", formule largement répandue dans les discours médiatiques contemporains (Ledoux, 2016). Ces impératifs se configurent comme le soubassement discursif sur lequel se fonde un discours explicite de sauvegarde du patrimoine européen, pour qu'il soit transmis aux générations futures :

"C'est un message de réconciliation qui doit nous habiter. À l'aube du XXI ${ }^{\mathrm{e}}$ siècle, l'actualité quotidienne ne nous invite-t-elle pas à réfléchir à la nécessité de privilégier l'échange pour une meilleure compréhension des individus entre eux ? Puisse donc le tourisme de mémoire contribuer à la rencontre entre générations du monde 
entier sur les terres de l'Aisne, de l'Oise et de la Somme.

En Picardie " (RP, 2013 : "Édito ", 5).

Les procédés énonciatifs et discursifs (construction présentative, emploi du déontique, question rhétorique, exhortation à l'impératif) ainsi utilisés permettent d'inscrire en discours une "éco-idéologie de la sauvegarde " (Paveau, 2009) qui embrasse la communauté entière ("nous"), dont les limites ne sont pas définies.

$\mathrm{Si}$, comme dans tout guide touristique (Moirand, 2004), la dimension didactique est également patente dans les guides Michelin, elle ne semble pas participer, du moins de manière aussi explicite, de la construction d'une communauté mémorielle européenne ; elle passe plutôt par l'adoption d'un point de vue, pourrait-on dire, surplombant, permettant de dépasser les clivages d'autrefois entre les peuples européens :

"La propagande a alors accusé les Allemands d'obliger la population civile à demeurer sur place afin de contraindre l'artillerie française à réduire ses bombardements. Mais la réciproque était vraie. On croyait simplement que la guerre serait courte" (MCD, 2014 :128).

Le " mais " à fonction adversative introduisant un jugement des auteurs sous forme de constat, l'emploi de l'indéfini " on " réunissant Allemands et Français dans le même espoir, et, enfin, le modalisateur " simplement " réduisant l'importance de l'enjeu conflictuel, ne sont que des exemples de moyens discursifs témoignant de la volonté de l'équipe éditoriale de prendre des distances par rapport aux discours insistant sur les clivages et les divisions, et de porter un regard plus objectif et neutre sur les faits de guerre. Les soldats, français et allemands, sont dès lors considérés en filigrane comme des victimes ayant subi la guerre, au-delà des antagonismes entre les gouvernements, éloignés de leurs véritables préoccupations sur les champs de bataille.

\section{La deuxième strate : la dimension collective et partagée}

L'héritage du passé ainsi patrimonialisé se charge de valeurs symboliques partagées par la communauté et dans les- 
quelles tout européen est susceptible de se reconnaitre : les événements tragiques du $\mathrm{XXI}^{\mathrm{e}}$ siècle permettraient de récupérer ces valeurs via la découverte des lieux de mémoire. Dans les guides, les descriptions des lieux de mémoire et de ce qu'ils offrent aux visiteurs témoignent de la volonté des rédacteurs de mettre en relation ces lieux avec une dimension collective européenne.

Autrefois lieux de conflits, aujourd'hui lieux symboles de la construction européenne, fondée sur la paix et la réconciliation entre les peuples : les lieux sont reconfigurés par le discours, qui en fait des objets discursifs susceptibles de cristalliser les valeurs fondatrices de l'Europe, au-delà des clivages dont ces mêmes lieux ont été les témoins lors des guerres mondiales. C'est bien le cas de l'extrait suivant, où l'accent est mis sur le rôle joué par le fort de Mutzig dans la construction de la paix européenne :

" La visite du fort propose une réflexion globale sur les guerres franco-allemandes qui, en quelques siècles, conduiront jusqu'aux deux guerres mondiales. Deux frères ennemis qui comprendront, dans l'après 1945, l'impossibilité de résoudre les crises par la guerre et décideront de créer les bases d'une Europe pacifique par la réconciliation franco-allemande " $(P F, 2016: 54)$.

La dénomination métonymique, presque oxymorique, " deux frères ennemis ", reformule les " guerres franco-allemandes " et permet de reconfigurer le passé "troublé " et de reconnaître au fort sa place dans le processus même de construction européenne. C'est bien l'Europe des valeurs de paix, de liberté et des droits de l'homme, tout comme le traité de Lisbonne l'a établi, qui sous-tend les extraits suivants :

"Sur les terres des batailles les plus meurtrières de la Première Guerre mondiale, ce centre est un lieu d'exposition, de réflexions et de rencontres au service de la promotion de la paix, des libertés et des droits de l'homme. Une exposition permanente intitulée " De la guerre à la paix " raconte la réconciliation franco-allemande, la construction européenne, la guerre dans l'histoire d'aujourd'hui, l'organisation des Nations unies et le souhait d'un monde en paix " (PF, 2016 : 147). 
"Classé monument historique en 1921, le HWK est aujourd'hui le site de mémoire de la Grande Guerre dédié à la paix entre les peuples le plus visité d'Alsace " (MAM, 2012 : 50 ; caractères gras des auteurs).

"Le monument aux morts est surprenant. Il illustre le relèvement de cette ville détruite, aspirant aux bienfaits d'une paix durable par l'union et la solidarité des peuples " (MCD, 2014 : 84).

Il peut arriver également que les auteurs recourent aux citations directes de figures historiques ayant contribué à forger l'Europe d'aujourd'hui, dans les descriptions des lieux, comme dans les extraits suivants :

"Une plaque sur l'esplanade rappelle la rencontre entre Mitterrand et Kohl de 1984, avec une pensée commune pour les morts des deux guerres mondiales. Ils ont déclaré : "Nous nous sommes réconciliés, nous nous sommes compris, nous sommes devenus amis" [nécropole nationale de Douaumont] "(RGG, 2015 : 150).

"Le 5 juin, le général Eisenhower, commandant en chef de l'opération, en rappelait les enjeux dans un message aux soldats qui s'apprêtaient à attaquer la "forteresse Europe" : "Soldats, marins et aviateurs des forces expéditionnaires alliées, vous êtes sur le point de vous lancer dans une grande croisade qui mobilise tous nos efforts depuis de longs mois. Le regard du monde est tourné vers vous. Les espoirs et les prières des hommes qui partout aiment la liberté vous accompagnent. En compagnie de nos alliés courageux et de nos frères d'armes sur d'autres fronts, vous amènerez la destruction de la machine de guerre allemande, l'élimination de la tyrannie nazie sur les peuples opprimés d'Europe et la sécurité pour nous tous dans un monde libre" "( $R D, 2014: 6)$.

Dans la citation d'Eisenhower choisi par Le Routard, l'Europe est présentée comme une entité unie déjà existante ("forteresse Europe "), menacée par les Allemands, et à défendre et préserver intacte contre "la tyrannie nazie". L'inscription en discours des paroles des "pères " de la construction européenne peut aussi se faire dans des encadrés constituant des digressions et des approfondissements typiques des guides Michelin :

" Anne Morgan (1873-1952), infatigable philanthrope

Elle est la FILLE du banquier John Pierpont Morgan, et la sœur d'un des acteurs principaux de la reconstruction 
européenne après la Grande Guerre. Connue pour son action en faveur de l'aide aux sinistrés en France, durant les deux guerres mondiales " (MCD, 2014 : 55).

"LA NAISSANCE DES "TOCH"

L'esprit de la Talbot House donne naissance à un mouvement d'entraide religieuse qui se développe juste après la guerre pour venir en aide aux soldats démobilisés, en s'inspirant de la "fraternité des tranchées" "(MFA, 2013 : 146).

La valorisation des lieux de mémoire passe aussi à travers différentes déclinaisons du topos de terre de résilience. Ce serait alors la capacité de ces régions à faire face aux évènements tragiques qui les ont ravagées qui permettrait aujourd'hui de sauvegarder ce patrimoine commun dont tout individu peut profiter : l'on assiste à la création en discours d'une forte identité territoriale qui entraîne tout un language of value (Kinney, 2017 : 105) contribuant à inscrire ces lieux dans une mémoire collective européenne, tout en restituant une image fortement valorisante des populations locales. Et cela est vrai surtout pour les Routard de la Picardie et de la Normandie :

"Dans la chaleur de l'été 1914, la Picardie devient le théâtre d'une guerre que tous pensaient courte et circonscrite, mais qui va s'avérer longue et embraser l'Europe entière " $(R P, 2013: 11)$.

" Un CONFLit INTERNATIONAL, UNE PAGE DE LA MÉmoire UNIVERSElLE Il n'est pas de paysage en Picardie qui ne possède une parcelle de la mémoire de la Grande Guerre. Dans la Somme, cette mémoire est plus qu'ailleurs partagée par les différents pays qui ont participé au conflit " $(R P, 2013$ : 73).

"Lors du $70^{\mathrm{e}}$ anniversaire de la bataille de Normandie en 2014, la Normandie tout entière se mobilise à nouveau pour honorer les derniers vétérans qui viennent se recueillir et se souvenir de ces moments historiques et de leurs "frères d'armes". Pour tous les Normands, c'est à nouveau l'occasion d'exprimer leur reconnaissance en commémorant cet événement historique qui a changé la face du monde. Au-delà des journées des 5 et 6 juin, cet anniversaire se déroule tout au long de l'année et sur tout le territoire, car il n'est pas une commune normande qui ne se sente concernée par cette histoire, "son histoire" : mais le $70^{\mathrm{e}}$ anniversaire de la bataille de Normandie marque 
incontestablement un tournant. La transition d'un tourisme du souvenir et de la commémoration vers un tourisme de mémoire va maintenant s'opérer. Le $70^{\mathrm{e}}$ anniversaire témoigne donc de la transmission de la mémoire et du partage de valeurs fondamentales : la paix, la réconciliation, la liberté auprès des nouveaux publics et, tout particulièrement, des jeunes générations " ( $R D, 2014$ : 155).

Ces extraits montrent la volonté de concilier une identité régionale, construite sur un imaginaire de résilience et de sacrifice des populations locales, avec une identité européenne englobant tous les pays ayant participé aux conflits, et de repositionner ces territoires dans une histoire européenne, voire mondiale.

\section{La troisième strate : la dimension émotive}

"L'émotion apparaît ainsi, pourrait-on dire, comme la preuve du patrimoine " (Heinich, 2013 : 195) : l'expression des émotions joue un rôle important dans le processus discursif de patrimonialisation, au point que certains chercheurs ont pu parler d'" émotions patrimoniales " (Fabre, 2013). Le discours touristique des guides analysés est parsemé d'émotions, ce qui constitue une tendance plutôt généralisée dans les textes touristiques (Rigat, 2017). Dans ces guides, les émotions participent de la construction en discours d'une mémoire "prothétique "(Raus, 2017 : xII), capable d'enclencher un jeu de projections et d'identifications entre les touristes potentiels et ceux qui ont vécu les traumatismes. Pour ce faire, les auteurs recourent volontiers à la mise en discours des émotions des soldats, saisis dans des moments de tristesse et de difficulté, parfois par l'expression des manifestations physiques d'émotions :

"Les Allemands, repliés sur leurs secondes lignes de défense, font des trouées sanglantes parmi les troupes britanniques, et l'on dit que certains mitrailleurs allemands pleuraient devant la facilité d'un tel carnage " (MCD, $2014: 34)$.

L'énonciation des émotions des soldats est accompagnée de la dramatisation du récit des événements. Cet effet de dramatisation est créé à partir de l'emploi de lexèmes (" san- 
glantes ", " carnage ") susceptibles de représenter de bons candidats à l'inscription en discours d'une "visée pathémique "(Plantin, 1998) diffuse, qui repose aussi sur des constructions syntaxiques particulièrement aptes à créer un effet pathémique (relatif à l'expression des passions), sans recourir forcément à des termes d'émotion. C'est le cas de l'exemple suivant, où l'" horrible guerre " est énoncée dans une question rhétorique feignant d'interpeller directement le lecteur :

"Pourquoi à Vauquois et dans les combats de l'Argonne à la fin du semestre de 1915, les deux adversaires ont-ils recours à l'horrible guerre des mines?" (MVAS, 2011 : 36).

Très souvent, les émotions sont rattachées à leurs "objets déclencheurs " et, par là, sont présentées comme des propriétés intrinsèques des lieux de mémoire décrits :

"Dans les vignes, une émouvante stèle marque l'endroit où sont tombés deux soldats du 66 $\mathrm{e}$ RI les 25 et 26 juillet 1918, au cours des journées où le hameau de Chassins a été repris maison par maison, à la grenade et au corps à corps" (MS, 2016 : 166).

" Le carrefour de la D 34 et de la D 64 offre une vision poignante du village disparu de Nauroy, rattaché à celui de Beine " (MMC, 2011 : 139).

"À l'entrée de chaque cimetière militaire, on trouve dans une boîte un registre avec l'état civil des soldats enterrés et un livre de signature pour les visiteurs. N'hésitez pas à les feuilleter. Très émouvant, car derrière chaque croix on devine un homme, souvent jeune, voire très jeune. Les témoignages des familles venues quelquefois de très loin se recueillir sur la tombe de leur ancêtre sont également très éloquents " $(R P, 2013$ : 76).

Le but est de faire partager une émotion de pitié pour les morts et les tragédies, en insérant dans le récit historiographique les émotions que les lecteurs sont susceptibles d'éprouver à la vue de ces lieux et de partager avec les soldats : les guides pointent là la dimension humaine du conflit, les soldats sont montrés en tant que sujets contraints à vivre une situation limite, éprouvant des émotions, plutôt dysphoriques, qui vont bien au-delà des contrastes entre les nations. 


\section{Conclusion}

Tous les guides de mémoire, qu'ils soient " pratiques " ou " culturels ", participent de la construction en discours d'un patrimoine mémoriel européen, supranational, fondé sur des valeurs identitaires européennes, bien que ce caractère européen se décline de manière un peu différente dans les deux typologies de guides. Si, en effet, les procédés discursifs mis en évidence permettent d'identifier dans l'ensemble du corpus différentes strates discursives entrelacées, liées à la nécessité de transmission du passé, au partage de ce passé par les citoyens européens, et, enfin, à la communion de valeurs et d'émotions censées être partagées par tout citoyen européen, les guides Michelin ne semblent pas adhérer au discours explicite liant les valeurs européennes au développement du tourisme de mémoire, et tiennent un discours qui se veut plus soucieux de rétablir une certaine précision historiographique. La dimension européenne du patrimoine est alors créée dans ces guides à travers la mise en récit d'épisodes de fraternisation entre les soldats des champs adverses et, plus en général, à travers l'insistance sur la dimension humaine du conflit, où les soldats sont saisis en tant qu'individus partageant un même destin tragique. Tout comme dans les discours officiels, les lieux de mémoire se configurent donc comme des lieux où s'est forgée l'identité européenne et, par là, ils sont censé véhiculer toutes les valeurs de paix et de réconciliation qui lui sont associées. Cela arrive également dans les guides " pratiques " qui, néanmoins, n'hésitent pas à tenir un discours patrimonial plus explicite. D'une part, ils pointent la dimension émotive à travers un effet de dramatisation des enjeux dont les lieux de mémoire seraient porteurs dans la société contemporaine. D'autre part, dans une perspective de promotion touristique, ils euphémisent les réalités dysphoriques liées aux passés troublés et insistent davantage sur la valorisation de la mise en tourisme des lieux décrits.

Il serait alors intéressant de creuser davantage ces tendances dans d'autres corpus, tels que les discours oraux des guides lors des visites, les guides d'autres pays ou les appli- 
cations mobiles de visite, afin de relever d'éventuelles différences dans la représentation des lieux de mémoire - selon les supports, la nationalité et l'âge du public ciblé.

Manuscrit reçu le 15 octobre 2019

Version révisée reçue le 26 septembre 2019

Article accepté pour publication le 7 octobre 2019

\section{Bibliographie}

Bertrand (Léon). 2004. «Déclaration de M. Léon Bertrand, ministre délégué au Tourisme, sur le tourisme de mémoire mis en exergue avec la célébration du $60^{\mathrm{e}}$ anniversaire du Débarquement ». En ligne : http://discours. vie-publique.fr/notices/043001108.html [vérifié le 16 octobre 2019].

Fabre (Daniel) (dir.). 2013. Émotions patrimoniales. Paris : Éd. de la Maison des sciences de l'homme (Ethnologie de la France).

Heinich (Nathalie). 2013. "Esquisse d'une typologie des émotions patrimoniales ", p. 195-210 in Émotions patrimoniales / sous la direction de Daniel Fabre. Paris : Éd. de la Maison des sciences de l'homme (Ethnologie de la France).

Davallon (Jean). 2006. Le Don du patrimoine. Une approche communicationnelle de la patrimonialisation. Paris : Hermes Science-Lavoisier.

Gellereau (Michèle). 2011. "Le récit de témoignage sur les usages comme reconstruction du sens des objets ». Culture \& Musées, 18, p. 75-97.

Hertzog (Anne). 2013. "Quand le tourisme de mémoire bouleverse le travail de mémoire ».Espaces, 313, p. 52-61.

Hertzog (Anne). 2012. "Tourisme de mémoire et imaginaire touristique des champs de bataille».Via@, 1. En ligne : https://journals.openedition.org/ viatourism/1276 [consulté le 16 octobre 2019].

Halbwachs (Maurice). 1967. La Mémoire collective. Paris : Presses universitaires de France (Bibliothèque de philosophie contemporaine).

Kerbrat-Orecchioni (Catherine). 2004. "Suivez le guide ! Les modalités de l'invitation au voyage dans les guides touristiques : l'exemple de l' "île d'Aphrodite" ", p. 133-150 in La Communication touristique, Approches discursives de l'identité et de l'altérité / sous la direction de Fabienne Baider, Marcel Burger \& Dionysis Goutsos. Paris : L'Harmattan.

Kinney (Hannah Wirta). 2017. "Antique sculpture, copies, and the language of value in early eighteenth-century grand tour guidebooks", p. 103-120 in Le Guide touristique : Lieu de rencontre entre lexique et images du patrimoine culturel / sous la direction de Rachele Raus, Gloria Cappelli \& Carolina Flinz. Florence : Firenze University Press. 
Kleiber (Georges). 1986. "Adjectif démonstratif et article défini en anaphore fidèle ", p. 169-185 in Les Déterminants : Syntaxe et sémantique / sous la direction de Jacques David \& Georges Kleiber. Paris : Klincksieck.

Kott (Christina). 2013. "L' "Exposition des œuvres d'art mutilées" de 1916 ", p. 119-145 in Émotions patrimoniales / sous la direction de Daniel Fabre. Paris : Éd. de la Maison des sciences de l'homme (Ethnologie de la France).

Kottelat (Patricia). 2015. "Du tourisme de pèlerinage au tourisme de mémoire : la réédition des guides des champs de bataille Michelin », p. 265276 in Dans l'amour des mots, Chorale(s) pour Mariagrazia / sous la direction de Paola Paissa, Françoise Rigat \& Marie-Berthe Vittoz. Alessandrie : Edizioni dell'Orso.

Ledoux (Sébastien). 2016. Le Devoir de mémoire. Une formule et son bistoire. Paris : CNRS Éditions.

Margarito (Mariagrazia). 2004. "Quelques configurations de stéréotypes dans les textes touristiques", p. 117-132 in La Communication touristique. Approches discursives de L'identité et de l'altérité / sous la direction de Fabienne Baider, Marcel Burger \& Dionysis Goutsos. Paris : L'Harmattan.

Moirand (Sophie). 2004. "Le même et l'autre dans les guides de voyage au $\mathrm{XXI}^{\mathrm{e}}$ siècle ", p. 151-173 in La Communication touristique. Approches discursives de l'identité et de l'altérité / sous la direction de Fabienne Baider, Marcel Burger \& Dionysis Goutsos. Paris : L'Harmattan.

Ministères de la Défense et du Tourisme. 2012. Le Tourisme de mémoire en France. Paris :Atout France.

Mainguenau (Dominique) \& Chareaudeau (Patrick). 2002. Dictionnaire d'analyse du discours. Paris : Seuil.

Nora (Pierre). 1984. "Préface " in Les Lieux de mémoire. La République / sous la direction de Pierre Nora. Paris : Gallimard.

Paveau (Marie-Anne). 2009. "La notion de patrimoine : lignées culturelles et fixations sémiotiques", p. 25-36 in Les Fictions patrimoniales sur grand et petit écran / sous la direction de Pierre Beylot \& Raphaëlle Moine. Bordeaux : Presses universitaires de Bordeaux.

Plantin (Christian). 1998. "Les raisons des émotions », p. 3-50 in Forms of Argumentative Discourse / sous la direction de Marina Bondi. Bologne : Clueb.

Raus (Rachele). 2017. "Préface", p. vII-XIV in Le Guide touristique : Lieu de rencontre entre lexique et images du patrimoine culturel / sous la direction de Rachele Raus, Gloria Cappelli \& Carolina Flinz. Florence : Firenze University Press.

Rigat (Françoise). 2017. "L'écrit de médiation à l'épreuve de l'interculturalité ", p. 105-128 in Scrittura e linguaggi. Viaggi tra parole, interpretazioni, esperienze / sous la direction de Laura Balbiani \& Dorit Kluge. Rome : Edizioni Nuova Cultura. 
Urbain (Jean-Didier). 2003. "Tourisme de mémoire. Un travail de deuil positif ». Cabiers Espaces, 80, p. 5-9.

\section{Sources et documents}

\section{Guides Michelin}

Les Champs de bataille. La Marne et la Champagne (2011).

Les Champs de bataille.Verdun, Argonne, Saint-Mibiel (2011).

Les Champs de bataille. Alsace Moselle : Les combats des Vosges (2012).

Les Champs de bataille. Flandres Artois : Ypres, Nord, Pas-de-Calais (2013).

Les Champs de bataille. Le Chemin des Dames :Aine, Oise (2014).

Les Champs de bataille. Somme :Amiens, Péronne, Albert (2016).

\section{Le Routard}

Picardie 14-18. Centenaire d'un conflit mondial (2013).

Le Débarquement \& la bataille de Normandie (2014).

Grande Guerre 14-18. Les chemins de mémoire. Des Flandres aux Vosges (2015).

Petit Futé

Guide des lieux de mémoire (2016). 


\section{Auteur}

\section{Stefano Vicari, Université de Gênes}

Professeur associé de langue et linguistique françaises à l'Université de Gênes (Italie), Stefano Vicari est spécialiste d'analyse du discours. Ses centres d'intérêt concernent principalement la linguistique populaire (ou folk linguistics) dans le domaine francophone, comme le témoigne son premier ouvrage Pour une approche de la linguistique populaire en France. Attitudes, prédiscours, questions de confiance, publié aux éditions Aracne (2016) et l'analyse des lettres des soldats français de la Grande Guerre, dans une perspective mémorielle. Actuellement, il participe à un projet du Département de langues et cultures étrangères de l'Université de Gênes (Italie) portant sur la mémoire collective européenne des passés troublés du $\mathrm{XX}^{\mathrm{e}}$ siècle.

Courriel : stefano.vicari@unige.it 


\section{Le patrimoine européen dans les guides touristiques de mémoire}

Cette étude, qui se situe dans la perspective méthodologique de l'analyse du discours, a pour objectif de relever les procédés discursifs à travers lesquels les guides touristiques de mémoire imprimés construisent un discours patrimonial de la mémoire européenne. À la suite de la présentation du tourisme de mémoire et des enjeux civiques et éthiques qui lui sont associés par les institutions et par les différents acteurs engagés dans le développement de ce type de tourisme, elle montre la construction de la dimension européenne du patrimoine constitué par les lieux de mémoire français. L'hétérogénéité des typologies de guides touristiques analysés (des guides culturels Michelin aux guides pratiques Le Routard et Petit Futé) permet d'observer un large éventail de procédés discursifs variés ainsi que de montrer les strates discursives à travers lesquelles se dessine au fil du discours le caractère " européen " du patrimoine mémoriel.

\section{Mots-clés}

tourisme de mémoire, patrimoine européen, guides touristiques, analyse du discours, discours touristique

\section{The European Heritage in the Tourist Guides of Memory}

Drawing on discourse analysis, as conducted in a French methodological perspective, this paper aims to identify the discursive procedures used in tourist guide books of memory to construct a heritage discourse of European memory. After introducing the "tourism of memory" as well as the related civic and ethical issues the institutions and the various actors involved assign to this type of tourism, the paper underlines the books' process of building the European dimension of French heritage sites seen as places of memory. The diverse range of guide book types covered (from the Michelin cultural guides to the practical Le Routard and Petit Futé) then allows to point a wide array of discursive procedures and layers gradually outlining the European character of these heritage sites.

\section{Keywords}

memory tourism, European heritage, tourist guidebooks, discourse analysis, tourist discourse 


\section{El patrimonio europeo en las guías turísticas de memoria}

Este estudio, que se sitúa dentro de la perspectiva metodológica francesa de análisis del discurso, tiene como objetivo detectar los procesos discursivos a través de los cuales las guías turísticas de memorias impresas construyen un discurso patrimonial de la memoria europea. Tras la presentación del turismo de la memoria y de los desafíos cívicos y éticos con los que las instituciones y los diferentes actores comprometidos con el desarrollo de este tipo de turismo les relacionan, se mostrará la construcción de la dimensión europea del patrimonio, constituido por los lugares de la memoria francesa, en las guías turísticas. La heterogeneidad de tipologías de las guías turísticas analizadas (de las guías culturales Michelin a las guías prácticas Le Routard y Petit Futé) permitirá observar un amplio abanico de procesos discursivos, mostrando al mismo tiempo los estratos discursivos a través de los cuales se dibuja a lo largo del discurso el carácter "europeo" del patrimonio de la memoria.

\section{Palabras clave}

turismo de la memoria, patrimonio europeo, guías turísticas, análisis del discurso, discurso turístico 